\title{
Differentiation of Using Flattening Filter Free Energy in Vmat Plans for Prostate Cancer
}

\author{
Yonca Yahşi Çelen ${ }^{1}$ and Atilla Evcin ${ }^{2}$ \\ ${ }^{1}$ Department of Radiation Oncology, University of Kocatepe, 03200, Afyon, Turkey \\ ${ }^{2}$ Department of Materials Science and Engineering, University of Kocatepe, 03200, Afyon, Turkey
}

\begin{abstract}
It is aimed to compare the values of conformity index (CI), homogeneity index (HI), monitor unit (MU) of volumetrically adjusted arthritis therapy (VMAT) plans using 10 prostate cancer patients with flattened filter (FF) and without flattening filter (FFF). In the study, treatment plans were prepared using $6 \mathrm{FF}$ and $6 \mathrm{FFF}$ in the Eclipse (ver.13.6) treatment planning system with Varian Trilogy Linear Accelerator. When planning was completed, CI averaged 0.87 , HI averaged 0.44 and MU values were found to be $591 \pm 26.8,650 \pm 33.06$, respectively. When the PTV coverage, $\mathrm{CI}, \mathrm{HI}$ and MU comparisons were made as a result of planning, there was no significant difference when comparing VMAT plans in FFF and FF energies. When we compare the MU values, the MU increase is seen when the straightening filter is removed. In both energy modes, good homogeneity in PTV was achieved with conventional francitation and close dose rates. No significant advantages and disadvantages of the unfiltered energy mode were observed in the assessment of plan quality in terms of CI, HI.
\end{abstract}

\section{Introduction}

Cancer disease is one of the most common health problems in recent years. Many patients with cancer have radiotherapy with or without chemotherapy. Radiotherapy is a method used in the treatment of cancer and some benign diseases using ionizing radiation.

The main goal in radiotherapy planning is to minimize the exposure of intact tissues to radiation while the target volume gives the necessary dose. Providing tumor control on this side also improves the quality of life as well as the prolongation of the life span $(1,2)$.

Prostate cancer is the most common cancer in men. Standard therapy Radiotherapy is performed locally and locally advanced. Due to the widespread use of prostate specific antigen (PSA) screening and the development of an ultrasound-guided biopsy technique, diagnosis can be made at an earlier stage than in the past (3). By applying RT and surgery, the disease at the local-advanced stage can be controlled at a high rate 
In recent years, advances in radiotherapy have resulted in conventional radiotherapy being replaced by Intensity Modulated Radiotherapy (IMRT) and Volumetric Arthritis Therapy (VMAT) techniques. These techniques provide better conformity to the target area and allow for the least possible intake of risky organs.

With IMRT and VMAT techniques, dose distribution can be obtained within the target volume with the radiotherapy fields or arc-based fixed control points arranged at different densities applied using non-uniform beam bundles. Multiple beam combinations at different intensities can be optimized to produce higher tumor control, lower normal tissue side effects. (4).

As shown in figure 3, another development in the technology is the use of standard linear accelerators without flat filter (FFF) heat. Expenditures are expected to decline in FFFs because of the reduced head scatter of accelerators. Thus, it is possible to reduce the dose of critical organ which is overdosed due to not being scattered, and the dose rate is higher treatment is performed in a shorter time.

It is aimed to compare the values of conformity index (CI), homogeneity index (HI), and monitor unit (MU) of volumetrically adjusted arc therapy (VMAT) plans using 10 prostate cancer patients with flattened filter (FF) and without flattening filter (FFF)

\section{Fundamental Properties of the Method}

CT images of 10 selected prostate cancer patients were scanned with $3 \mathrm{~mm}$ cross section and transferred to TPS. Using these CT images, target structures were plotted by gross tumor volume (GTV) and clinical target volume (CTV), planned target volume (PTV), and critical organs of radiation oncology. Patient plans were prepared using Varian Trilogy Linear Accelerator with 6 MV FF and 6 MV FFF in Eclipse (ver.13.6) treatment planning system. As shown in figure 1, two-tiered VMAT plans have been adopted as standard. Two backed VMAT plans with gantry turns in opposite directions were created. The plans were aimed at minimizing the leakage and "tongue and groove" effect from the MLCs with $30^{\circ}$ and $330^{\circ}$ collimator angles on opposite sides of the arcs. 6FF and 6FFF plans were made for each patient. As shown in figure 2, after optimization and calculations, the maximum doses of target and critical organs of each plan were examined with the help of DVH. The target and OAR maximum dose values, homogeneity and conformality of PTV and MU values were compared for FF and FFF plans.

Conformity index (CI) was developed as part of dosimetric analysis. When $\mathrm{CI}$ is equal to 1 , ideal conformfality is achieved. The conformity index greater than 1 means that the irradiated volume is larger than the target volume and contains healthy tissue. If the conformity index is less than 1 , only a part of the target volume is irradiated. The conformality index equation is given below (5).

$\mathrm{CI}=\mathrm{VRI} / \mathrm{TV}$

$\mathrm{VRI}=$ irradiated volume $(\mathrm{cc}), \mathrm{TV}=$ tumor volume $(\mathrm{cc})$

The conformity index values of the target volume of the PTV in the plans made using this equation are calculated and compared for FFF and FF beams.

The dose homogeneity characterizes the absorptive dose distribution in the target volume. Homogeneity index equal to or close to 0 indicates that the absorptive dose distribution is homogeneous. The HI equation is given below (6).HI=( D\%2 - D\%98)/ D\%50

D $2 \%=$ target dose of $2 \%$ volume, D $98 \%=$ target dose of $98 \%$ volume,

D $50 \%=$ the dose of the target $50 \%$ of the volume

Using this equation, the homogeneity index values of the target volume of the PTVs are calculated for the FFF and FF beams. 


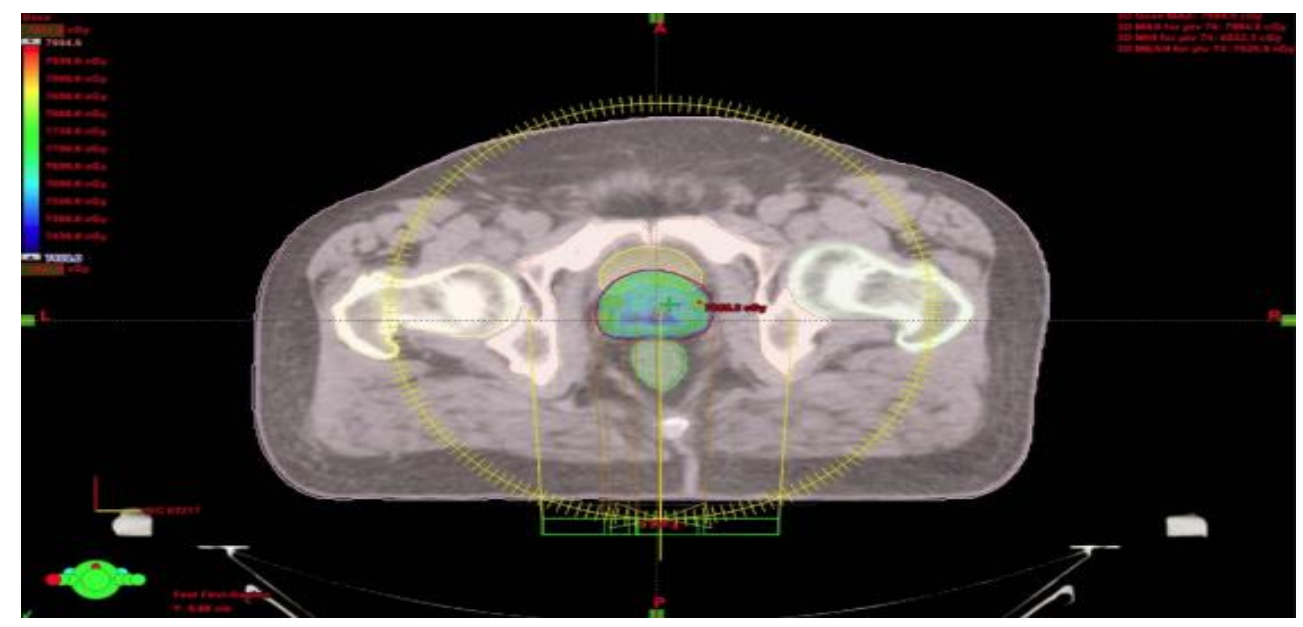

Fig.1. Treatment areas of planned prostat cancer patient with VMAT technique.

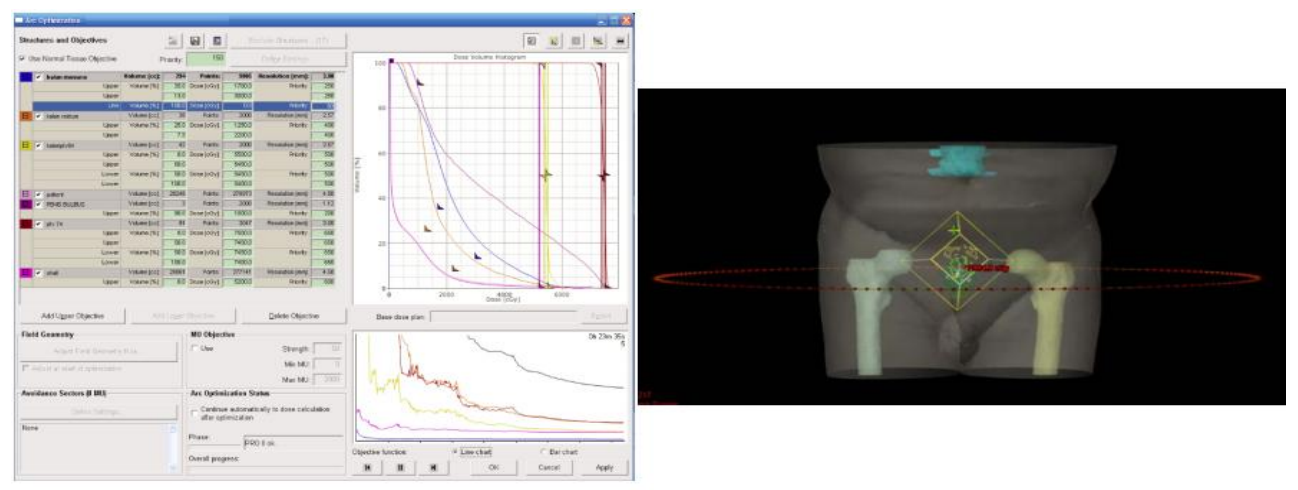

Fig.2. Optimization window and dose volume histogram

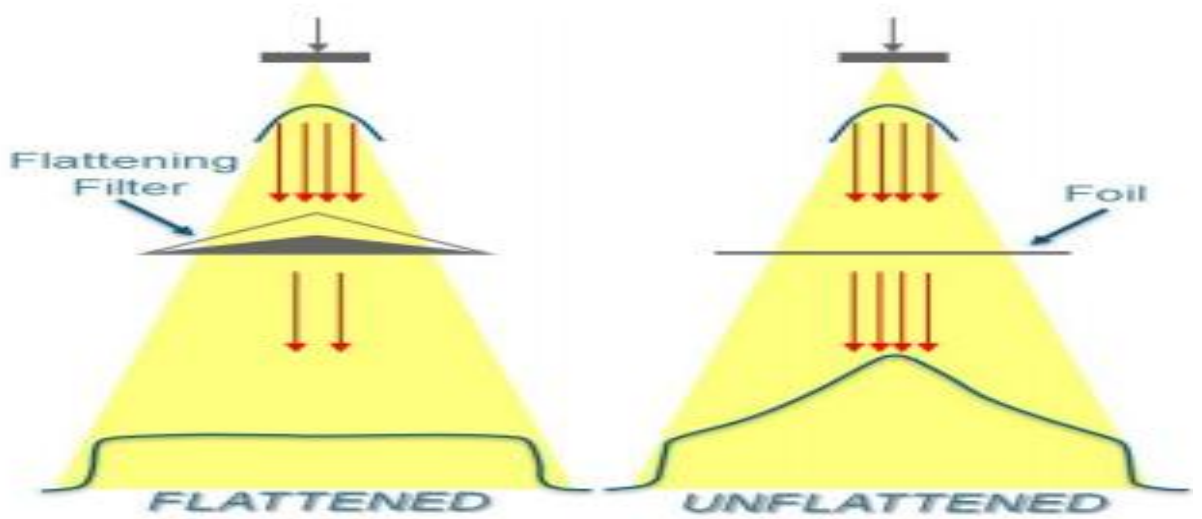

Fig.3. Physical structure in FFF energy 


\section{Application}

As shown in figure 4, when the plans were completed, the mean $\mathrm{CI}$ for the FF and FFF plans was 0.87 , the mean $\mathrm{HI}$ was 0.44 , and the MU values were found to be $591 \pm 26.8,650$ \pm 33.06 , respectively. As a result of planning, when PTV coverage, CI, HI and MU comparisons were made, there was no significant difference when comparing VMAT plans for FFF and FF energy. When we compare the MU values, the MU increase is seen when the straightening filter is removed.

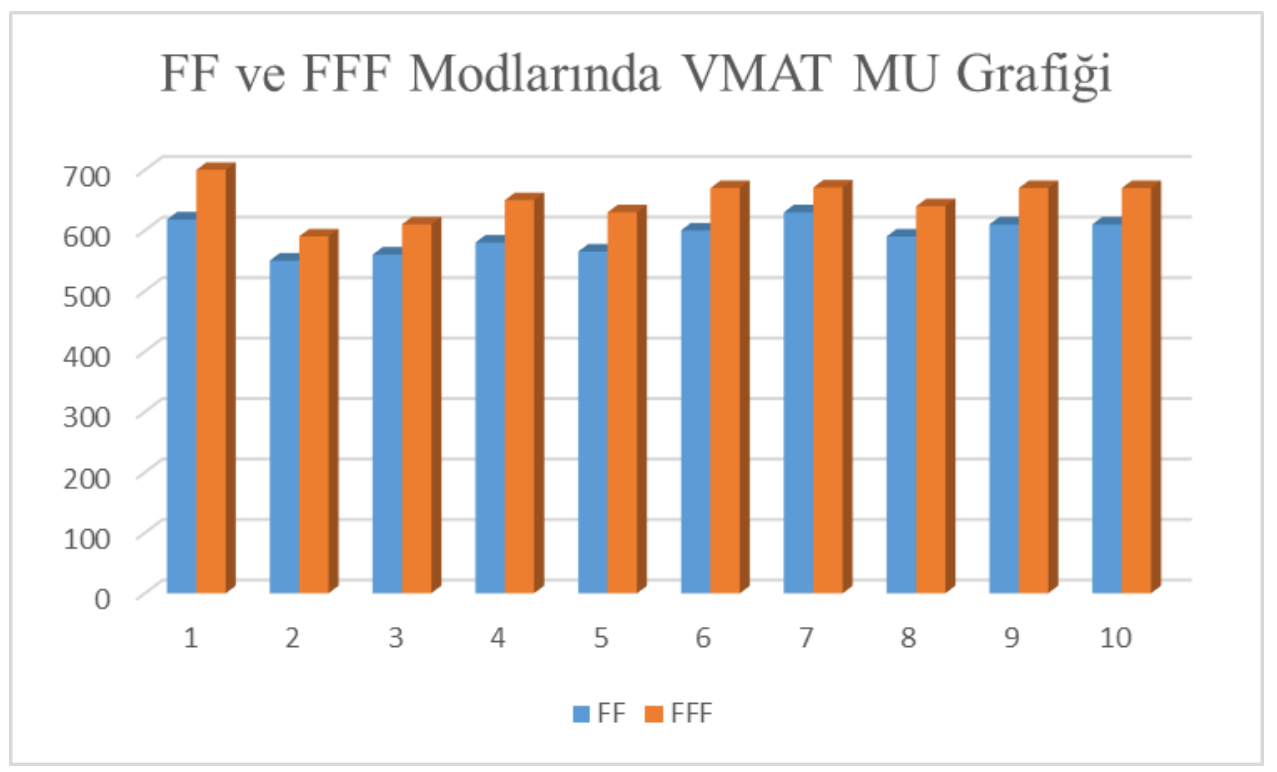

Fig.4. VMAT MU graph in FF and FFF modes

\section{Conclusion}

There has recently been an interest in the clinical use of liners (FFF) where there is no straightening filter. As a result of removing the smoothing filter; significant increase in dose rate, softening in photon flux (thus decreased in penetration), reduction in head scatter (thus reduction in peripheral dose) and uneven open beam profile. Other important features of the FFF linacs are that the surface dose and the maximum dose shift slightly to the surface. Increased dose rate for FFF linac; the energy of the photon beam, the linac maker, and the field size. The dose rate factor varies from 2 to 4 depending on these conditions. With increased dose rate, the duration of treatment is reduced, the likelihood of target motion is minimized, treatment volume may increase, and potentially lead to radiobiological benefits for some tumors (7). In addition, FFF linear accelerators usually have fewer neutron generations. Uncorrected rays with these positive features can reduce the risk of secondary cancer (8).

When FF is applied and not implemented, PTV has reached its target dose and critical organs at risk have been obtained within the initially defined dose limits. 
Compared with the dose distribution effect of the use of the smoothing filter, the hot doses in the PTV target volumes were found to be significantly larger than those applied in the FF-unplanned schemes; but this difference is limited to $1 \%$.

Zwahlen et al. In patients with prostate cancer, using $10 \mathrm{MV}$ scans, it was reported that in the plans they performed with the VMAT technique, target homogeneity increased not $1 \%$ but not significantly when FF was not applied. Zwahlen et al. (9) reported an increase of $24.7 \%$ in the total MU value in the plans made with non-FF beams.

Ong et al. reported an $8.3 \%$ increase in the total MU value in the plans made with non-FF radiation. (10) The increase in the total MU value in this study is due to Zwahlen et al. and Ong et al. However, because Ong et al. Finds a lesser increase, the target volume may be smaller than this study. The reason for this increase in the total MU value is that the reduction of the flattening filter in unfused beams reduces the dose to the edge of the field relative to the FF rays; so that the FFF rays can be further modulated by the IMRT fields to increase the MU value so that the different dose levels occurring between the central region and the peripheral region can be homogenized. This increase in MU will increase the normal tissue integral dose (NTID). The increase in MU was found to be an expected result in order to make the dose levels homogeneous.

In both energy modes, good homogeneity in PTV was achieved with conventional francitation and close dose rates. No significant advantages and disadvantages of the unfiltered energy mode were observed in the assessment of plan quality in terms of CI, HI. When we compare the MU values, the MU increase is seen when the straightening filter is removed.

\section{References}

1. K. S. Chao, C. A. Perez, L. W. Brady, Nobel Tıp Kitabevleri, İstanbul, (2004)

2. F. M. Khan, The Physics of Radiation Therapy, 3, (2003)

3. C. Öbek, Türkiye Kanserle Savaş Vakfı Onkolojide Güncel Yaklaşım Toplantıları, 23-29 (2011)

4. A. Çakır, F. Ağaoğlu, B. Şahin, M. Okutan, Y. Dizdar, E. Darendeliler, Türk Onkoloji Dergisi 24(4), 166-171 (2009)

5. L. Feuvret, G. Noel, J. J. Mazeron, P. Bey, Rad.. Oncol. Biol. Phys 64(2), 333-342 (2006)

6. International Comission on Radiation Units and Measurements (ICRU). Report 83

Prescribing, Recording and Reporting Photon Beam Intensity Modulated Radiation Therapy (IMRT) 2010.

7. P. Tsiamas, J. Seco, Z. Han, M. Bhagwat, J. Maddox, C. Kappas, K. Theodorou, M.

Makrigiorgos, K. Marcus, P. Zygmanski, Med Phys 38(5), 2342-2352 (2011)

8. Y. Wang, M. K. Khan, J. Y. Ting, S. B. Easterling, Rad.. Oncol. Biol. Phys, 83(2), 281285 (2012)

9. D. R. Zwahlen, S. Lang, J. Hrbacek, C. Glanzmann, S. Kloeck, Y. Najafi, T. Streller, G. Studer, K. Zaugg, Rad. Oncol. Biol. Phys 83(5), 1655-1660 (2012)

10. C. L. Ong, W. F. Verbakel,M. Dahele, J. P. Cuijpers, B. J. Slotman, Rad.. Oncol. Biol. Phys. 83(1), 137-143 (2012) 